である.いま $H_{\infty_{0}}=1 ， c_{m 2_{\infty}}=1$ になるような尺度で 特性曲線を畫くと旸根數無限の犲根車に對しては特性 曲線は點 $(0 ， 1) ，(1 ， 0)$ を通る直線となる。これに對 し牙根數有限のものは點 $\left(0, h_{0}\right) ，\left(c_{m}, 0\right)$ を通る值線 となる.こっに

$$
h_{0}=k_{1}, \quad c_{m}=k_{1} / k_{2}
$$

また無衝突流入は點 $\left(c_{e}, h_{e}\right)$ で與えられる.こつに

$$
c_{e}=k_{3} / k_{4}, h_{e}=\left(k_{1} k_{4}-k_{2} k_{3}\right) / k_{4}
$$

以上の結果からつぎのことが結論できる.

$k_{1} ， k_{3}$ は $n_{2} \lambda$ のみの函數， $k_{2}, k_{4}$ は $n, \lambda$ と牙 根の形狀 $f\left(\xi^{\prime}\right) / f(\lambda / 2)$ の函數であるが狗根の出口角 $\beta_{2}$ には無關係である.したがつて $h_{0}, c_{m}, h_{e}, c_{e}$ は $\beta_{2}$ に無關係である。

この旸根車特性曲線の一般な性質は Busemannの 對數渦卷線の犲根車に對する計算結果においても成立 している.第 4 圖は Busemann の論文の圖より求め たもので $\beta_{2}$ に對する $h_{0}, h_{e}, c_{m}$ は $\beta_{2}=90^{\circ}$ 附近 において $\beta_{2}$ に無關倸である.

註：一

(1) 昭和 21 年 7 月 13 日關西支部第 147 回講演 曾において講演，原稿受付昭利 21 年 7 月 25 日.

(2) 正員, 京都帝國大學.

(3) W. Spannhake, Hydraulische Probleme, 1926. A. Busemann, Z.A.M.M., Bd. 8, 1928.

\title{
.翼列理 論につつい妾
}

藤 本 武 助 ${ }^{(2)}$ 廣 瀨 幸 “治 $^{(3)}$

\section{描 要}

この論文は翼列の理論について述べたもので 2 部より成立つ. 第 1 部は一檔でない流れの中にある 翼に對し Munk の薄翼の理論を症張したものであり，第2 部は第 1 部の考え方を直線翼列に適用し た結果で任意翼型の翼列の計算法を示した。第 1 部は第 1 の著者により，第 2 部は第 1 の著者のす めにより第 2 の著者が計算した結果である。

茅 I 部 一樣でない流え の中の翼

1. 埪界條件之循環の決定

翼は略本面板に近く，かつそれが $x$ 軸上一1より +1 の間にあるも のとする.すなわち翼弦長を 2 とと る.また翼がない場合に翼上の速度 の $x ， y$ 分速庭を并れぞれ $V+u ，$

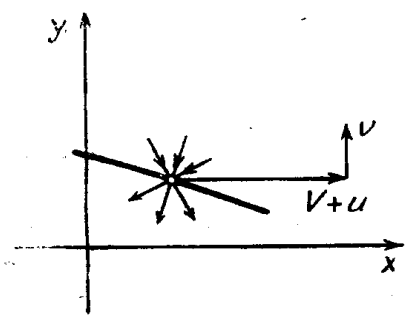

第 1 図

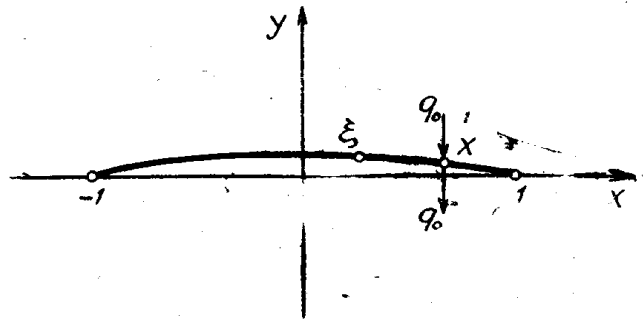

第 2 圆 $v$ とする.こっで $V$ は一定の値， $u, v$ は場所によ り異なるが $V$ にくらべて小さいとする.

翼がこの流れの中に置かれた場合に翼面上の境界條 件を滿足させるため䝷面上に吹出，吸込を分布させる. 翼面上單位長さについての吹出の强さを $q$ とすると $x$ 點の $d x$ の部分よりの吹出の强さは $q d x$ となる. $q d x$ と翼面上 $d x$ の部分を贵いて流れる流體量を等し くおけば $q$ が求まる.すなわち

$$
\begin{aligned}
& q d x=-(V+u)(d y / d x-v / V) d x, \\
& q=-(V+u) d y / d x+v
\end{aligned}
$$

翼の下面にこれだけの吹出を置くと同時に翼上面には 同じ强さの吸込を置 。

翼面上の點 $x$ で下面に $q_{0}$ の吹出，上面に $q_{0}$ の吸 込を置いた場合 $\xi$ 點における速度は

$$
u_{q}(\xi)= \pm \frac{q_{0}}{\pi(x-\xi)} \sqrt{\frac{1-x^{2}}{1-\xi^{2}}}
$$

ただし＋は上面に， 一 は下面にとる.

$x に q d x$ の吹出, 吸込がある場合の $\xi$ の速度を $d u_{q}(\xi)$ とすると

$$
d u_{q}(\xi)= \pm \frac{q d x}{\pi(x-\xi)} \sqrt{\frac{1-x^{2}}{1-\xi^{2}}}
$$

翼全體に互つて吹出，吸込が分布したときの $\xi の$ 速度 $u_{q}(\xi)$ は 


$$
u_{q}(\xi)= \pm \frac{1}{\pi} \int_{-1}^{+1} \frac{q}{x-\xi} \sqrt{\frac{1-x^{2}}{1-\xi^{2}}} d x
$$

ーよより與えられる。

さて兾の後緣すなわち $\xi=1$ の附适についで考える に $\xi=1-\varepsilon$ とおくと

$$
u_{q}(1-\varepsilon)=\mp \frac{1}{\pi \sqrt{2 \varepsilon}} \int_{-1}^{+1} q \sqrt{\frac{1+x}{1-x}} d x
$$

となり，もヤ0ににおいて無限大をなる。そこで KuttaJoukowski，の條件を滿足させるために裔のまわりの 循瞻 $\Gamma$ の流れを附加するに時針方向のを正とすると 菑面上の速度は，

$$
u_{\Gamma}(\xi)= \pm \frac{\Gamma}{2 \pi \sqrt{1-\xi^{2}}}
$$

$$
\begin{aligned}
\xi=1-\varepsilon \text { では } \\
u_{\Gamma}(1-\varepsilon)= \pm \frac{\Gamma}{2 \pi \sqrt{2 \varepsilon}}
\end{aligned}
$$

(3), (5) の二つの和を $u^{\prime}$ とすると

$$
u^{\prime}= \pm \frac{1}{\sqrt{2 \varepsilon}}\left\{\frac{\Gamma}{2 \pi}-\frac{1}{\pi} \int_{-1}^{+1} q \sqrt{\frac{1+x}{1-x}} d x\right\}
$$

$\varepsilon \rightarrow 0$ で $u^{\prime}=0$ の條件により

$$
\Gamma=2 \int_{-1}^{+1} q \sqrt{\frac{1+x}{1-x}} d x
$$

となり，翼のまわりの循環 $\Gamma$ の大きさは決定する: あるいは

$$
\Gamma=2 \int_{-1}^{+1} \sqrt{\frac{1+x}{1-x}}\left(-V \frac{d y}{d x}-u \frac{d y}{d x}+v\right) d x
$$

\section{2. 循理の分布, カおよびモーメント}

買面上の循摆の分布を $\gamma(\xi)$ と表わすと

$$
\begin{aligned}
r(\xi) & =\frac{\Gamma}{\pi \sqrt{1-\xi^{2}}}+2 u_{q}(\xi) \\
& =\frac{\Gamma}{\pi \sqrt{1-\xi^{2}}}+\frac{2}{\pi} \int_{-1}^{+1} \frac{q}{x-\xi} \sqrt{\frac{1-x^{2}}{1-\xi^{2}}} d x
\end{aligned}
$$

これより翼にはたらく力の $x ， y$ 方向の分力をそれぞ れ $P_{x}, P_{y}$ とすると

$$
\begin{aligned}
P_{y} & =\rho \int_{-1}^{+1} \gamma(\xi)(V+u) d \xi \\
& =\frac{\rho \Gamma}{\pi} \int_{-1}^{+1} \frac{V+u}{\sqrt{1-\xi^{2}}} d \xi \\
& +\frac{2 \rho}{\pi} \int_{-1}^{+1}(V+u) d \xi \int_{-1}^{+1} \frac{q}{x-\xi} \sqrt{\frac{1-x^{2}}{1-\xi^{2}}} d x
\end{aligned}
$$

$$
\begin{aligned}
P_{x} & =-\rho \int_{-1}^{+1} \gamma(\xi) v d \xi \\
& =-\frac{\rho \Gamma}{\pi} \int_{-1}^{+1} \frac{v}{\sqrt{1-\xi^{2}}} d \xi \\
& -\frac{2 \rho}{\pi} \cdot \int_{-1}^{+1} v d \xi \int_{-1}^{+1} \frac{q}{x-\xi} \sqrt{\frac{1-x^{2}}{1-\xi^{2}}} d x
\end{aligned}
$$

また座標軸原點すなわち翼弦中央の點のまわりのモー メントは

$$
\begin{aligned}
& M=\frac{\rho \Gamma}{\pi} \int_{-1}^{+1} \frac{(V+u) \xi}{\sqrt{1-\xi^{2}}} d \xi \\
& +\frac{2 \rho}{\pi} \int_{-1}^{+1}(V+u) \xi d \xi \int_{-1}^{+1} \frac{q}{x-\xi} \sqrt{\frac{1-x^{2}}{1-\xi^{2}}} d x
\end{aligned}
$$

以上一漛でない流れの中の翼についで得られた結果 は直緗および圓形黛列の場合にも應用できる，すなわ ち他の翼を渦または渦の分布により表わし，それによ る $u ， v$ を求めると (6)，(8)，(9)，(10)により $\dot{\Gamma}$, 力, モーメントは決定する。これは䨢列訊算の一方法 であるが第 2 部ではこの部で考えた Munkの方法を 直接直線䔬列に適用することを試みた。

\section{3. 厚ささ影帮亡計等例}

翼厚の影響を考えに入れるには兾表面の $x$ 軸に對 する傾斜を二つの部分に゙分ち

$$
d y / d x=(d y / d x)_{0}+(d y / d x)_{t}
$$

とする. $(d y / d x)_{e}$ は反りによる部分, $(d y / d x)_{t}$ は唇さ による部分であるーこの $(d y / d x)_{t}$ に鷹ずる吹出，罗 込を兾面に沿い分布すればよい。

例えばＶの速度の一梳流れの中に反りのない兾を 置いた場合には翼表面の境界條件を滿足するためには $V(d y / d x)_{t}$ の吹出を $x$ の上下兩面に置けばよい.

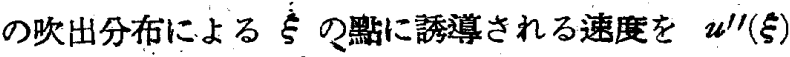
とすると

$$
u^{\prime \prime}(\xi)=-\frac{V}{\pi} \int_{-1}^{+1} \frac{(d y / d x)_{t}}{x-\xi} d x
$$

により與えられる.反りのある翼の場合には 1 師で求 まる厚さのない目面上の速度にびを附加えればよい。 つぎに計算例を二、堨ずる。

$u=a x ， v=-a y(a$ は定數) の流れの中に测角 $\alpha$ の本面板がある場合を考えよう. 暻面の形はy=- $\alpha x$ で $d y / d x=亡 \alpha$ ，また $v=-a y=a \alpha x$ である.よつ $\tau(6) に よ り$

$$
\begin{array}{r}
\Gamma=2 \int_{-1}^{+1}(\nu \alpha+\alpha \alpha x) \sqrt{\frac{1+x}{1-x}} d x \\
+2 \int_{-1}^{+1} a \alpha x \sqrt{\frac{1+x}{1-x}} d x=2 \pi \alpha(V+\alpha)
\end{array}
$$

また

$$
\begin{aligned}
& P_{y}=2 \pi \rho V^{2} \alpha-\pi \rho V a a_{s} \\
& P_{x}=\pi \rho V a \alpha^{2}
\end{aligned}
$$


となる・

つぎに一溙流れの中に反りのない翼のある場合を考 える. $x=-\cos \theta$ の置換をなし; 翼表面の形 $y_{t}$ が $y_{t}=b_{1} \sin \theta+b_{2} \sin 2 \theta+\cdots \cdots+b_{n} \sin n \theta+\cdots$

で表わされるとすると

$(d y / d x)_{t}=\left(b_{1} \cos \theta+\cdots \cdots+n b_{n} \cos n \theta\right) / \sin \theta$

したがつて翼面上の速度 $u^{\prime \prime}$ は (11)により $\xi=-\cos \theta_{0}$ とすると

$$
\begin{aligned}
u^{\prime \prime} & =\frac{V}{\pi} \int_{0}^{\pi} \frac{\sum n b_{n} \cos n \theta}{\cos \theta-\cos \theta_{0}} d \theta \\
& =V \sum n b_{n} \frac{\sin n \theta_{0}}{\sin \theta_{0}}
\end{aligned}
$$

これは等角舁像による嚴密な解法の第一近似值と一致 する.

\section{第 II 部 直線翼列の理論}

\section{4. 平面板列の等角富像}

第 3 圖の如く $z$ 面で喰堂、角 $\gamma$, ピッチ $d$ の平面 板の直線墨列を考え，これを头式の關係によりと面の 單位圓に倪像する。

$$
z=\frac{d}{2 \pi}\left\{e^{-i \gamma} \log \frac{\kappa \zeta+1}{\kappa \zeta-1}+e^{i r} \log \frac{\zeta+\kappa}{\zeta-\kappa}\right\}^{(4)}
$$

$\zeta$ 面の單位圓の周上 $\zeta=e^{i \theta}$ に對しては

$$
\begin{aligned}
& x= \frac{d}{\pi}\left\{\cos \gamma \tanh ^{-1} \frac{2 \kappa \cos \theta}{1+\kappa^{2}}\right. \\
&\left.+\sin \gamma \tan ^{-1} \frac{2 \kappa \sin \theta}{1-\kappa^{2}}\right\}+n d \sin \gamma, \\
& y \text { 흐 } n d \cos \gamma, n=0, \pm 1, \pm 2, \ldots \ldots \ldots
\end{aligned}
$$

前緣 $A$, 後緣 $B$ に對應する $\theta$ をそれぞれ $\theta_{L}, \theta_{T}$ とすれば

$$
\tan \theta_{L}=\tan \theta_{T}=\frac{1-\kappa^{2}}{1+\kappa^{2}} \tan \gamma
$$

したがつて冥弦長を $c$ ，ピッチ弦辰比を $\lambda$ とすると

$$
\begin{aligned}
\frac{1}{\lambda}= & \frac{c}{d}=\frac{2}{\pi}\left\{\cos \gamma \tan h^{-1} \frac{2 \kappa \cos \gamma}{\sqrt{\kappa^{4}+2 \kappa^{2} \cos 2 \gamma+1}}\right. \\
& \left.+\sin \tan ^{-1} \frac{2 \kappa \sin \gamma}{\sqrt{\kappa^{4}+2 \kappa^{2} \cos 2 \gamma+1}}\right\} \ldots \ldots(15)^{\prime}
\end{aligned}
$$

\section{5. 吹出，吸込分布および循環による流れ}

$z$ 面で翼の上下面に吸込，欥出を分布した場合の速 度を求める。

兾面上の一點 $x に q_{0}$ なる吹出を置いた場合，他の 黠 $x_{0}$ に導き出される速度を $u$ とする． $x ， x_{0}$ に對 應する圆上の點を $\zeta \equiv e^{i \theta}, \zeta_{0}=e^{i \theta_{0}}$ とすると $\zeta$ 面の 数素ポテンシャルは

$$
W_{1}=\frac{q_{0}}{\pi} \log \left(\zeta-e^{i \theta}\right)-\frac{q_{0}}{2 \pi} \log \zeta
$$

$u=\left(\frac{d W}{d \zeta} \frac{d \zeta}{d z}\right)_{\zeta=\zeta_{0}}$ でこれは計算の結果つぎのよう になる。

$u=-\frac{q_{0}}{4 d \kappa} \cdot \frac{\kappa^{4}-2 \kappa^{2} \cos 2 \theta_{0}+1}{\left\{\left(1+\kappa^{2}\right) \cos \gamma \sin \theta_{0}-\left(1-\kappa^{2}\right) \sin \gamma \cos \theta_{0}\right\}}$

$$
\frac{\sin \left(\theta_{0}-\theta\right)}{1-\cos \left(\theta_{0}-\theta\right)}
$$

したがつて翼下面に欧出を分布させた場合に導き出 される速度は（16）を積分することにより得られる。 この場合吹出, 吸込分布の $z$ 面, $\zeta$ 面における值の 間には

$$
q(x) d x=q(\theta) d \theta
$$

の關係がある。こ〉に $q(x), q(\theta)$ はそれぞ始 $z$ 面， ら面の分布の强さを表わす。あるいは

$q(\theta)=-q(x) \frac{2 d \kappa\left\{\left(1+\kappa^{2}\right) \cos \gamma \sin \theta-\left(1-\kappa^{2}\right) \sin \gamma \cos \theta\right\}}{\kappa^{4}-2 \kappa^{2} \cos 2 \theta+1}$

したがつて㻎下面の吹出分布による $x_{0}$ 點つ速度を $u_{q 1}\left(\theta_{0}\right)$ と記號すると
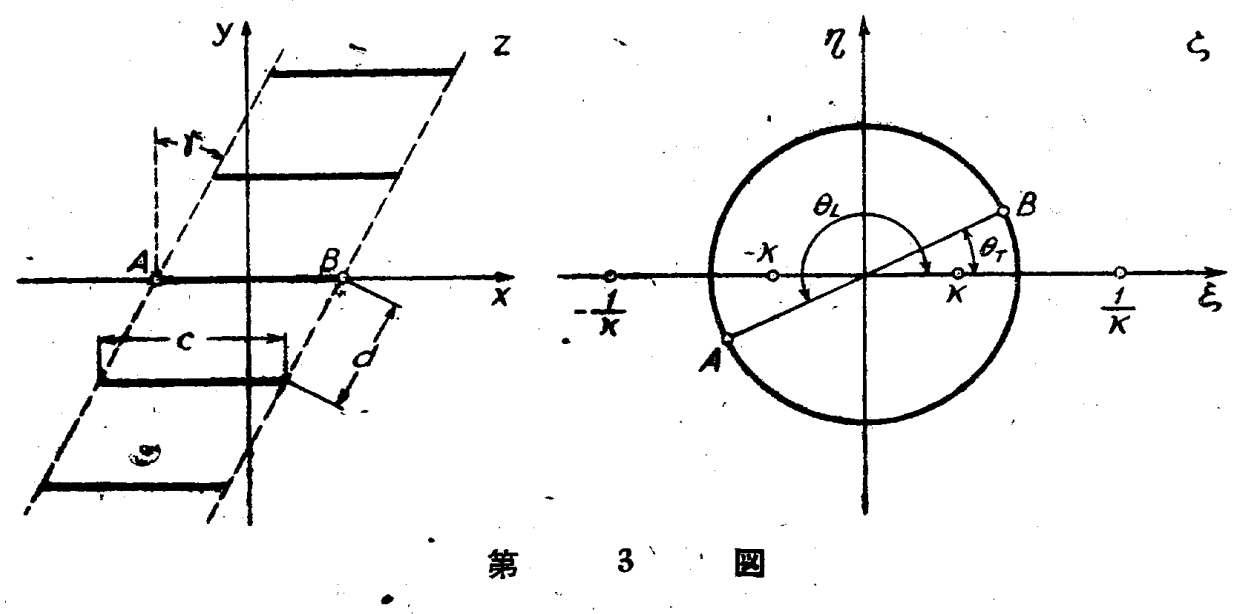


$$
\begin{aligned}
u_{q 1}\left(\theta_{0}\right)= & \frac{\kappa^{4}-2 \kappa^{2} \cos 2 \theta_{0}+1}{2 \pi\left\{\left(1+\hbar^{2}\right) \cos \gamma \sin \theta_{0}-\left(1-\kappa^{2}\right) \sin \gamma \cos \theta_{0}\right\}} \cdot \\
& \quad \int_{\theta \boldsymbol{T}+\pi}^{\theta \tau+2 \pi} q(x) \frac{\left\{\left(1+\kappa^{2}\right) \cos \gamma \sin \theta-\left(1-\kappa^{2}\right) \sin \gamma \cos \theta\right\}}{\kappa^{4}-2 \kappa^{2} \cos 2 \theta+1} \cdot \frac{\sin \left(\theta_{0}-\theta\right)}{1-\cos \left(\theta_{0}-\theta\right)} d \theta .
\end{aligned}
$$

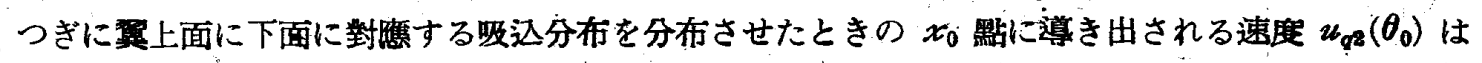

$$
\begin{aligned}
u_{q^{2}}\left(\theta_{0}\right)= & \frac{\kappa^{4}-2 \kappa^{2} \cos 2 \theta_{0}+1}{2 \pi\left\{\left(1+\kappa^{2}\right) \cos \gamma \sin \theta_{0}-\left(1-\kappa^{2}\right) \sin \gamma \cos \theta_{0}\right\}} \cdot \\
& \int_{\theta T}^{\theta T+\pi} q(x) \frac{\left\{\left(1+\kappa^{2}\right) \cos \gamma \sin \theta-\left(1-\kappa^{2}\right) \sin \gamma \cos \theta\right\}}{\kappa^{4}-2 \kappa^{2} \cos 2 \theta+1} \cdot \frac{\sin \left(\theta_{0}-\theta\right)}{1-\cos \left(\theta_{0}-\theta\right)} d \theta
\end{aligned}
$$

したがつて翼下面に吹出。上面に樶込を分布させた場合の速度 $u_{q}\left(\theta_{0}\right)$ は上の 2 式より

$$
\begin{aligned}
& u_{q}\left(\theta_{0}\right)= \frac{\kappa^{4}-2 \kappa^{2} \cos 2 \theta_{0}+1}{2 \pi\left\{\left(1+\kappa^{2}\right) \cos \gamma \sin \theta_{0}-\left(1-\kappa^{2}\right) \sin \gamma \cos \theta_{0}\right\}} \\
& \quad \int_{\theta T}^{\theta T+2 \pi} q(x) \frac{\left\{\left(1+\kappa^{2}\right) \cos \gamma \sin \theta-\left(1-\kappa^{2}\right) \sin \gamma \cos \theta\right\}}{\kappa^{4}-2 \kappa^{2} \cos 2 \theta+1} \cdot \frac{\sin \left(\theta_{0}-\theta\right)}{1-\cos \left(\theta_{0}-\theta\right)} d \theta
\end{aligned}
$$

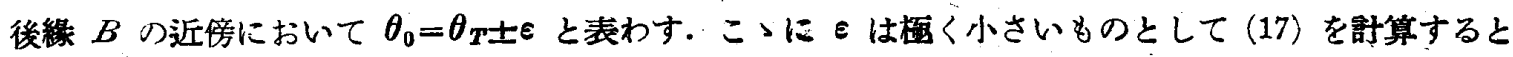

$$
u_{q}\left(\theta_{T} \pm \varepsilon\right)=\mp \frac{\left(1-\kappa^{4}\right)^{2}}{2 \pi \varepsilon\left(\kappa^{4}+2 \kappa^{2} \cos 2 \gamma+1\right)^{4}} \int_{\theta T}^{\theta T+2 \pi} q(x) \frac{1+\cos \left(\theta_{T}-\theta\right)}{\kappa^{4}-2 \kappa^{2} \cos 2 \theta+1} d \theta
$$

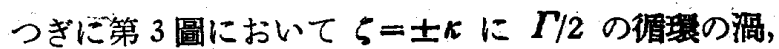
$\zeta= \pm 1 / \kappa に ー \Gamma / 2$ の渦をおくと $\zeta$ 面の單位圆のま わりの循環 $F$ の流れが得られる.この流れの 6 面に お放素ポテンシャルは

$$
W_{2}=\frac{i \Gamma}{4 \pi} \log \frac{\zeta^{2}-\kappa^{2}}{\zeta^{2}-1 / \kappa^{2}}
$$

したがつて $\theta_{0}$ に對應する䔬面上の遇度を $\dot{u}_{\Gamma}\left(\theta_{0}\right)$ とすると $u_{\Gamma}\left(\theta_{\theta}\right)=\left(\frac{d W_{2}}{d \zeta} \frac{d \zeta}{d z}\right)_{\zeta=\tau_{0}} に よ り$ $u_{\Gamma}\left(\theta_{0}\right)=\frac{\Gamma\left(1-\kappa^{4}\right)}{4 d \kappa\left\{\left(1+\kappa^{2}\right) \cos \gamma \sin \theta_{0}-\left(1-\kappa^{2}\right) \cdot \sin \gamma \cos \theta_{\theta}\right\}}$

後継 $B$ の近謗においては

$$
u_{\Gamma}\left(\theta_{T} \pm \varepsilon\right)= \pm \frac{\Gamma\left(1-\kappa^{4}\right)}{4 d \kappa \varepsilon \sqrt{\kappa^{4}+2 \kappa^{2} \cos 2 \gamma+1}} \ldots
$$

\section{6，循環およびカ}

前笁で得られた如く後線附近においては $u_{q}, u_{\mathrm{r}}$ は

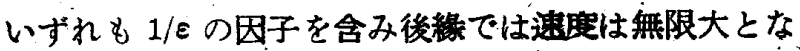

る. Kutta-Joulkowski の條件を满足するには (18);

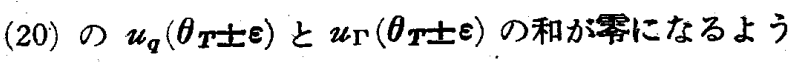

にすればよい.ょつて

$$
\begin{aligned}
\Gamma= & \frac{2 d \kappa\left(1-\kappa^{4}\right)}{\pi \sqrt{\kappa^{4}+2 \kappa^{2} \cos 2 \gamma+1}} \\
& \int_{\theta \boldsymbol{T}}^{\theta+2 \pi} q(x) \frac{1+\cos \left(\theta_{T}-\theta\right)}{\kappa^{4}-2 \kappa^{2} \cos 2 \theta+1} d \theta
\end{aligned}
$$

となる, こメで翼列に對する流れの本均速度をw，迎角すな わち $z$ 面の $x$ 軸と $w$ のなす角を $\alpha$ とすると

$$
q(x)=w \sin \alpha-w \cos \alpha \cdot d y / d x
$$

したがつて兾列の一曋に作用するカ $P$ は流體の密 度を $\rho$ とすると

$$
\begin{aligned}
& P=\rho \Gamma w^{\prime} \\
& \text { さらに } \quad(\rho / 2) w^{2} C_{L} c \text { と表わすと } \\
& . C_{L}=\frac{8 \lambda \kappa \sin \alpha}{\sqrt{\kappa^{4}+2 \kappa^{2} \cos \gamma+1}}-\frac{4 \lambda \kappa\left(1-\kappa^{4}\right) \cos \alpha}{\pi \sqrt{\kappa^{4}+2 \kappa^{2} \cos 2 \gamma+1}} \cdot \\
& \int_{\theta \boldsymbol{T}}^{\theta \pi+2 \pi} \frac{d y}{d x} \frac{1+\cos \left(\theta_{T}-\theta\right)}{\kappa^{4}-2 \kappa^{2} \cos 2 \theta+1} d \theta \quad \ldots(23)
\end{aligned}
$$

\section{7. 簡覃な形狀の要}

平面板の場合は $y=0, d y / d x=0$ で $C_{L}$ は $(23)$ 右 第 1 項のみとなる

扰物緗型の暴の場合に反りを $f$ とすると

$$
\begin{aligned}
& -y=\left(4 f / c^{2}\right)\left\{\left(c^{2} / 4\right)-f^{2}\right\} \\
& d y / d \dot{x}=-8 f x / a^{2}
\end{aligned}
$$

この場合（23）の右邊第 2 項の積分は

$$
\begin{aligned}
& \frac{32 \lambda^{2} \kappa f\left(1-\kappa^{4}\right) \cos \alpha}{\pi^{2} c \sqrt{\kappa^{4}+2 \kappa^{2} \cos 2 \gamma+1}} \\
& \quad \int_{\theta \boldsymbol{T}}^{\theta_{\boldsymbol{T}}+2 \pi} \varphi(\theta) \frac{1+\cos \left(\theta_{\mathrm{T}}-\theta\right)}{\kappa^{4}-2 \kappa^{2} \cos 2 \theta+1} d \theta
\end{aligned}
$$

$こ>に \varphi(\theta)=\cos \gamma \tanh ^{-1} \frac{2 \kappa \cos \theta}{1+\kappa^{2}}+\sin \gamma \tan ^{-1}$ $\frac{2 \kappa \sin \theta}{1-\kappa^{2}}$ である。 
いま $\varphi(\theta)=\sum a_{n} \cos n \theta+\sum b_{n} \sin n \theta$ と表わすと $n$ の偶數に對し

$$
\int_{0}^{2 \pi} \frac{\cos n \theta}{\kappa^{4}-2 \kappa^{2} \cos 2 \theta+1} d \theta=\frac{2 \pi \kappa^{n}}{1-\kappa^{4}}
$$

$n$ の奇數に對しては積分值は零，また被積分函數の分 子が $\sin n \theta$ のときも零であるから

$$
\begin{gathered}
a_{2 m+1}=\frac{2 \kappa^{2 m+1} \cos \gamma}{2 m+1}, \quad b_{2 m+1}=\frac{2 \kappa^{2 m+1} \sin \gamma}{2 m+1}, \\
m=0,1,2, \ldots \ldots
\end{gathered}
$$

故に $\int_{\theta \boldsymbol{T}}^{\theta \boldsymbol{T}+2 \pi} \varphi(\theta) \frac{1+\cos \left(\theta_{\boldsymbol{T}}-\theta\right)}{\kappa^{4}-2 \kappa^{2} \cos 2 \theta+1} d \theta$

$$
\begin{aligned}
& =2 \pi\left(\frac{\cos \gamma \cos \theta_{T}}{1-\kappa^{2}}+\frac{\sin \gamma \sin \theta_{T}}{1+\kappa^{2}}\right) \sum \frac{\kappa^{4 m+1}}{2 m+1} \\
& =\frac{\pi \sqrt{\kappa^{4}+2 \kappa^{2} \cos 2 \gamma+1}}{\kappa\left(1-\kappa^{4}\right)} \log \frac{1+\kappa^{2}}{1-\kappa^{2}}
\end{aligned}
$$

故に $C_{L}=\frac{8 \lambda \kappa \sin \alpha}{\sqrt{\kappa^{4}+2 \kappa^{2} \cos 2 \gamma+1}}$

$$
+\frac{22 \lambda^{2} f \cos \alpha}{\pi c} \log \frac{1+\kappa^{2}}{1-\kappa^{2}}
$$

\section{8. 任意翼方場合の計算方針}

任意翼の場合にも（23）の積分を求めねばならない。 その方法としてつぎのような一つの方法が考えられる。 $\theta^{\prime}=\theta_{\mathrm{T}}-\theta$ とし， $\theta_{\mathrm{T}}$ を基淮にして $\zeta$ 面の單位圆 の周を $4 p$ に等分し Simpson の法則を適用する. $\theta_{n}^{\prime}=n \pi / 2 p(n=0,1,2, \cdots \cdots)$ において

$$
h_{n}=\frac{1+\cos \theta_{n}^{\prime}}{\kappa^{4}-2 \kappa^{2} \cos 2\left(\theta_{T}+\theta^{\prime}{ }_{n}\right)+1}
$$

また $\theta^{\prime}{ }_{n}$ に對萑する點の $-d y / d x$ を一 $(d y / d x)_{n}$ と L

$$
f_{n}=-(d y / d x)_{n} h_{n}
$$

と表わすと

$$
\begin{aligned}
C_{L} & =\frac{8 \lambda \kappa \sin \alpha}{\sqrt{\kappa^{4}+2 \kappa^{2} \cos 2 \gamma+1}} \\
& +\frac{\lambda \kappa\left(1-\kappa^{4}\right) \cos \alpha}{9 \sqrt{\kappa^{4}+2 \kappa^{2} \cos 2 \gamma+1}}\left(2 \sum_{s=0}^{2 p-1} f_{2 s}+4 \sum_{s=0}^{2 p-1} f_{2 s+1}\right)
\end{aligned}
$$

により與えられる。 $h_{n}$ は $\gamma$ と入の函數である故, 種マの $\gamma, \lambda$ に對し豫め計算しておけばよい.

$p=6, \gamma=30^{\circ}, \lambda=1$ の場合について上のような計 算法がどれ程嚴密解に近いかを計算例により示すと扡 物線型の窝に對し

嚴密解 (24) 式 $C_{L}=4 \cdot 19 \sin \alpha+10.26 \frac{f}{c} \cos \alpha$,

$$
\text { 計算值 } \quad C_{L}=4 \cdot 19 \sin \alpha+10 \cdot 10 \frac{f}{c} \cos \alpha
$$

つぎに翼厚の影響を考えるに第 1 部で述べたと同样 $1=$

$$
d y / d x=(d y / d x)_{c}+(d y / d x)_{t}
$$

の三つの部分に分割する。 $(d y / d x)_{t}$ に對して翼上に分 布した吹出, 吸込分布に上り後線 $B$ 附近に生ずる速 度を $\boldsymbol{u}_{\boldsymbol{q}}{ }^{\prime}$ とすると

$$
\begin{gathered}
u_{q^{\prime}}\left(\theta_{T \pm \varepsilon)}=\mp \frac{\left(1-\kappa^{4}\right)^{2} w}{2 \pi \varepsilon\left(\kappa^{4}+2 \kappa^{2} \cos 2 \gamma+1\right)} \cdot\right. \\
\left\{\int_{\theta T}^{\theta T+\pi}\left(-\frac{d y}{d x}\right)_{t} \frac{1+\cos \left(\theta_{T}-\theta\right)}{\kappa^{4}-2 \kappa^{2} \cos 2 \theta+1} d \theta\right. \\
\left.\quad-\int_{\theta T+\pi}^{\theta T+2 \pi}\left(-\frac{d y}{d x}\right)_{t} \frac{1+\cos \left(\theta_{T}-\theta\right)}{\kappa^{4}-2 \kappa^{2} \cos 2 \theta+1} d \theta\right\} \\
\text { これに隹じて } C_{L} に \\
\Delta C_{L}=\frac{4 \lambda \kappa\left(1-\kappa^{4}\right) \cos \alpha}{\pi \sqrt{\kappa^{4}+2 \kappa^{2} \cos 2 \gamma+1}} I
\end{gathered}
$$

の變化を生ずる.Iは（27）・の積分の值である・さら に $\theta_{n^{\prime}}$ におけ施る一 $(d y / d x)_{t}$ を一 $(d y / d x)_{t n}$ とし

$$
f_{n}^{\prime}=-(d y / d x)_{t n} h_{n}
$$

とすると

$$
\begin{aligned}
\Delta C_{L} & =\frac{\lambda \kappa\left(1-\kappa^{4}\right) \cos \alpha}{9 \sqrt{\kappa^{4}+2 \kappa^{2} \cos 2 \gamma+1}}\left\{2\left(\sum_{s=0}^{p-1} f_{2 s}^{\prime}-\sum_{p+1}^{2 p+1} f_{2 s}\right)\right. \\
& \left.+4\left(\sum_{0}^{p-2} f_{2 s+1}^{\prime}-\sum_{p-1}^{2 p-1} f_{2 s+1}^{\prime}\right)\right\} \quad \ldots \ldots \ldots \ldots \ldots(28)
\end{aligned}
$$

となる。

終りに本呼究の第 1 部は服部報公會の援助をうけた 研究の一部であり，また文部省科學研究費の援助をう け，大塚記念獎學會の援助をうけたことを記し謝意を 表する.

\section{. 萑考文 献}

M, Munk, Fundamentals of Fluid Dynamics for Aircraft Designers.

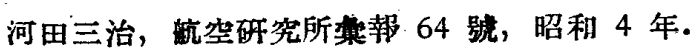
W.F. Durand, Aerodynamic Theory, Vol. II.

註 : -

（1）昭和 21 年 9 月 20 日關西支部第 148 回講演會 において講演，原稿受附昭和 21 年 11 月 7 日.

（2）正員，京都帝國大學.

（3）准員，京都帝國大學.

(4) $z=\frac{\alpha}{2 \pi}\left\{e^{-i \Upsilon \log } \frac{1+\kappa \zeta}{1-\kappa \zeta}+e^{i r} \log \frac{\zeta+\kappa}{\zeta-\kappa}\right\}$ の方か よいようであるが，䊅果にはなんら㱍はない。 (第 1 の著者誌). 